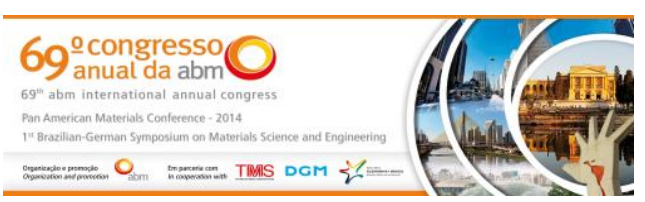

Tema: Metalurgia do Pó

\title{
EFEITO DA MOAGEM DE ALTA ENERGIA NA DENSIFICAÇÃO E MICROESTRUTURA DO COMPÓSITO $\mathrm{Al}_{2} \mathrm{O}_{3}-5 \% \mathrm{Cu}$ CERÂMICO DE MATRIZ METÁLICA*
}

\section{Resumo}

$\mathrm{O}$ compósito metal-cerâmico $\mathrm{Al}_{2} \mathrm{O}_{3}-\mathrm{Cu}$ alia as propriedades de baixa densidade, alta resistência ao desgaste, dureza e biocompatibilidade da fase cerâmica alumina a excelente condutividade elétrica, e alta tenacidade do cobre metálico. Tal compósito pode ser aplicado como ferramenta de corte, entre outras. Este trabalho discute a influência da moagem de alta energia na microestrutura e densificação do compósito $\mathrm{Al}_{2} \mathrm{O}_{3}-\mathrm{Cu}$. Pós de $\mathrm{Al}_{2} \mathrm{O}_{3}$ e $\mathrm{Cu}$ na razão de $5 \%$ em massa de Cu foram moídos por até 100 horas em moinho planetário de alta energia. A razão em massa de pó para bola usada foi de 1:5. O álcool etílico foi usado como solvente. Na moagem, amostras de pós foram colhidas após 2, 10, e 50 horas. Pós moídos foram prensados uniaxialmente em matriz cilíndrica a $100 \mathrm{MPa}$ e sinterizados a $1.300^{\circ} \mathrm{C}$ por 1 hora sob atmosfera de $\mathrm{Ar}$ e $\mathrm{H}_{2}$. A taxa de aquecimento foi de $10^{\circ} \mathrm{C} / \mathrm{min}$. Análises de $\mathrm{DRX}$, AG caracterizaram os pós moídos. O MEV e o EDS foi empregado para observar a microestrutura das partículas dos pós moídos e dos corpos sinterizados. Um pequeno tamanho de partícula da fase cerâmica e uma estrutura mais homogênea são produzidos quando os pós foram moídos até 100 horas.

Palavras-Chave: Moagem de alta energia; Sinterização; Compósito metal-cerâmico; Compósito $\mathrm{Al}_{2} \mathrm{O}_{3}-\mathrm{Cu}$.

\section{EFFECT OF HIHG ENERGY BALL MILLING ON DENSIFICATION AND MICROSTRUCTURAL OF COMPOSITE $\mathrm{Al}_{2} \mathrm{O}_{3}-5 \%$ Cu CERAMIC METAL MATRIX Abstract}

The metal - ceramic composite $\mathrm{Al}_{2} \mathrm{O}_{3}$ - Cu combines the properties of low density, high wear resistance, toughness and biocompatibility of the ceramic phase alumina excellent electrical conductivity, and high toughness of metallic copper. This composite can be used as cutting tools, among others. This paper discusses the influence of high energy milling on microstructure and densification of the composite $\mathrm{Cu}$ - Al2O3. Powders of $\mathrm{Al}_{2} \mathrm{O}_{3}$ and $\mathrm{Cu}$ in the ratio of $5 \% \mathrm{Cu}$ were milled for up to 100 hours of high-energy planetary mill. The mass ratio of powder to ball used was 1:5. Ethanol was used as solvent. In grinding, the powder were taken after 2,10 , and 50 hours. Milled powders were uniaxially pressed in a cylindrical die at $100 \mathrm{MPa}$ and sintered at $1,300^{\circ} \mathrm{C}$ for 1 hour under $\mathrm{Ar}$ and $\mathrm{H}_{2}$. The heating rate was $10^{\circ} \mathrm{C} / \mathrm{min}$. XRD, AG analysis, characterized the milled powders. The SEM and EDS was employed to observe the microstructure of the particles of the milled powders and sintered bodies. A small particle size of the ceramic phase and a more homogeneous structure are produced when the powders were milled to 100 hours.

Keywords: High-energy milling; Sintering; Metal-ceramic composite; Composite $\mathrm{Cu}-\mathrm{Al}_{2} \mathrm{O}$.

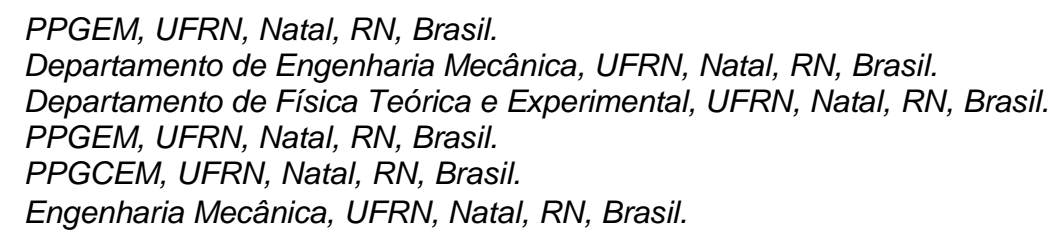

* Contribuição técnica ao 69 Congresso Anual da ABM - Internacional e ao 14ํㅡㄹ ENEMET - Encontro Nacional de Estudantes de Engenharia Metalúrgica, de Materiais e de Minas, 21 a 25 de julho de 2014, São Paulo, SP, Brasil. 
1 INTRODUÇÃO

Inovadoras aplicações tecnológicas necessitam de materiais que se caracterizam por combinações de propriedades que não podem ser identificadas individualmente em metais ou ligas metálicas convencionais, materiais cerâmicos ou poliméricos. De acordo com o princípio da ação combinada, melhores propriedades são criadas através da combinação de dois ou mais materiais distintos [1]. Materiais compósitos são compostos por duas fases, uma é matriz, que é continua e envolve a outra fase, chamada frequentemente de fase dispersa. As propriedades dos compósitos são funções das propriedades das fases de seus constituintes, das suas quantidades relativas e da geometria da fase dispersa. Entende-se como geometria da fase dispersa a forma da partícula, seu tamanho, sua distribuição e sua orientação.

Podem-se classificar os materiais compósitos com relação a sua matriz, podendo ser chamado de compósito com matriz polimérica (CMP), compósito com matriz cerâmica (CMC) e compósito com matriz metálica (CMM). O uso extensivo de compósitos de matriz metálica na indústria aeroespacial, indústria automotiva e em aplicações estruturais tem aumentado ao longo últimos 20 anos, devido à disponibilidade de reforços e rentáveis rotas de processamento que dão origem a propriedades reprodutíveis [2].

Nesta perspectiva observa-se que na confecção de materiais alternativos tem-se, por exemplo, que estruturas reforçadas podem ser obtidas com a união de partículas de um óxido cerâmico duro à uma matriz metálica dúctil. A partir de sua microestrutura, os materiais reforçados por partículas podem ser divididos em dois grupos: micro-compósitos e nano-compósitos. Denomina-se micro- compósitos os materiais em que as partículas são maiores que $1 \mu \mathrm{m}$ e nano-compósitos aqueles em que as partículas são menores que $100 \mathrm{~nm}$ [3]. Neste contexto, devido às dimensões extremamente pequenas, os materiais nanoestruturados apresentam grande fração volumétrica de contornos de grãos, proporcionando assim ganho nas propriedades mecânicas dos produtos obtidos [4]. As principais vantagens desses materiais são a alta homogeneidade da microestrutura aliada à alta sinterabilidade a temperaturas mais baixas. Isto produz materiais com melhores propriedades mecânicas.

A alumina é hoje um dos materiais cerâmicos mais utilizados na engenharia em decorrência de suas propriedades, tais como o elevado módulo de elasticidade, a alta refratariedade, a alta dureza, a alta resistência ao ataque químico, a alta resistência sob alta temperatura e a elevada rigidez dielétrica. Entretanto, sabe-se que o uso de uma única fase cerâmica produz materiais de uso limitado devido a sua alta fragilidade e baixa tenacidade à fratura.

Em relação ao cobre sabe-se que é um material excelente para aplicações elétricas cujo eficiência pode ser melhorada, melhorando as suas propriedades mecânicas [5]. Quando as partículas de alumina são dispersas na matriz de cobre, eles apresentam características únicas, como a alta condutividade térmica e elétrica, bem como uma elevada resistência e excelente resistência a recozimento [6].

A Alumina (Al2O3) tem uma densidade de $3,98 \mathrm{~g} / \mathrm{cm} 3$, um módulo de Young de 380 $\mathrm{GPa}$, excelente resistência à oxidação e de alta temperatura boas propriedades mecânicas [7-8]. O Cobre $(\mathrm{Cu})$ tem uma densidade de 8,92 g/cm3, um módulo de Young de $130 \mathrm{GPa}$ e uma boa resistência à fratura [8]. Assim, uma microestrutura consistindo de $\mathrm{Cu}$ e $\mathrm{Al} 2 \mathrm{O} 3$ pode ser capaz de fornecer propriedades como uma boa

* Contribuição técnica ao 69ํ Congresso Anual da ABM - Internacional e ao 14ํㅡㄹ ENEMET - Encontro Nacional de Estudantes de Engenharia Metalúrgica, de Materiais e de Minas, 21 a 25 de julho de 2014, São Paulo, SP, Brasil. 


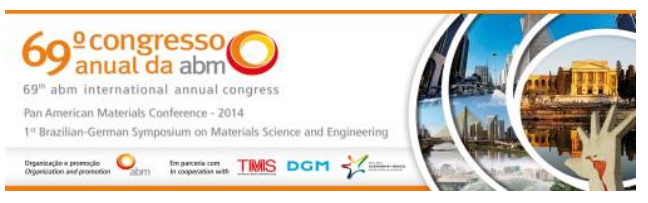

resistência à oxidação a alta temperatura e aos critérios de propriedades mecânicas do material estrutural de alta temperatura com sucesso.

Os pós nanométricos podem ser obtidos por vários métodos, dentre estes a moagem de alta energia (MAE) se destaca por permitir obter pós compósitos nanocristalinos no estado sólido [9]. Esta técnica difere dos outros tipos de moagem pela alta taxa de transferência de energia envolvida no processo causada pela elevada velocidade e frequência de impacto dos copos de moagem contra as partículas de pós. Isto produz uma intensa redução no tamanho de partícula, dispersão das fases, solubilidade sólida de uma fase na outra bem como pode amorfizar o material.

O processamento de pós por moagem de alta energia permite uma mistura a nível atômico possibilitando a produção de compostos nanocristalinos [10]. Este trabalho tem por objetivo investigar o efeito da dispersão de partículas de alumina na fase dúctil de cobre através da moagem de alta energia de pós compósitos $\mathrm{Al}_{2} \mathrm{O}_{3}-\mathrm{Cu}$ com o teor de Cobre ditado em $5 \%$.

\section{MATERIAIS E MÉTODOS}

A pesquisa foi desenvolvida como mostra a Figura 1 abaixo, em que inicialmente pode-se prepara o pó-compósito.

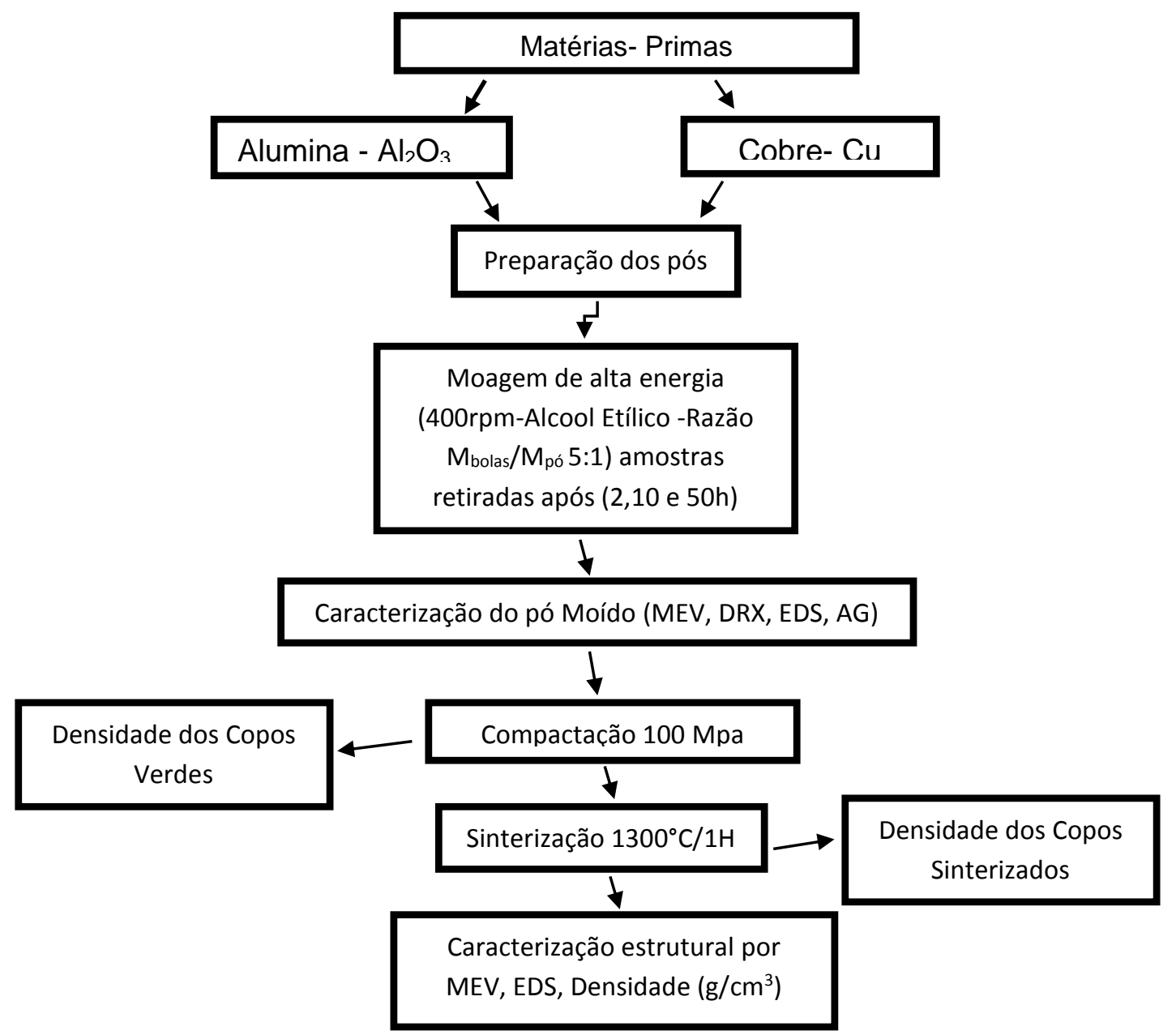

Figura 1. Esquema experimental empregado na preparação e obtenção do pó-compósito $\mathrm{Al}_{2} \mathrm{O}_{3}$ $5 \% \mathrm{Cu}$.

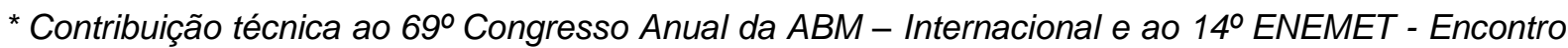
Nacional de Estudantes de Engenharia Metalúrgica, de Materiais e de Minas, 21 a 25 de julho de 2014, São Paulo, SP, Brasil. 


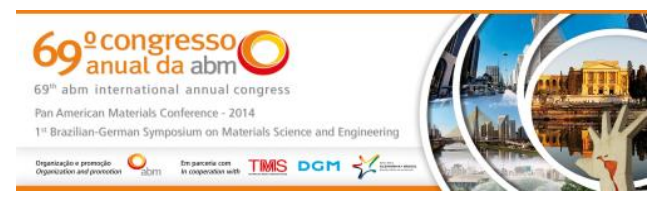

Para obter o pó compósito $\mathrm{Al}_{2} \mathrm{O}_{3}-5 \% \mathrm{Cu}$, uma carga de pó com $5 \%$ em massa de $\mathrm{Cu}$ foi moída em um moinho planetário Pulverisatte 7 de alta energia por até 100 horas. Após a preparação do pó realizou-se a caracterização do mesmo, em seguida o pó foi prensado a frio [11] em matriz cilíndrica em prensa uniaxial a $100 \mathrm{Mpa}$, obtendose ao final duas amostras compactadas ou corpos verdes.

A Sinterização dos corpos verdes é a etapa que sucede a compactação. Nesta fase utilizou-se um forno resistivo sob vácuo com atmosfera controlada com dois gases inertes Argônio e Hidrogênio segundo metodologia adaptada de Motta [11]. Deste modo os compactos de pós verdes foram sinterizados a $1300^{\circ} \mathrm{C}$ durante 60 minutos programando-se uma taxa de aquecimento de $10^{\circ} \mathrm{C} / \mathrm{min}$.

Após obter-se as amostras sinterizadas pode-se medir a densidade destes corpos, assim como a densidade dos corpos verdes obtidos antes da sinterização. Tal técnica pode ser efetuada através do método geométrico.

Os pós das estruturas sinterizadas foram analisadas através do, MEV, EDS e DRX e AG (Análise granulométrica).

\section{RESULTADOS E DISCUSSÃO}

Na Difração de Raios-X das amostras moídas do pó-compósito $\mathrm{Al}_{2} \mathrm{O}_{3}-5 \% \mathrm{Cu}$ os pós reagentes $\mathrm{Cu}$ e $\mathrm{Al}_{2} \mathrm{O}_{3}$, foram detectas (Figura 2). De modo comparativo, as quatro amostras dos pós, dentre elas, os pós puros e moídos relacionados, pode-se visualizar a presença de picos relativos aos pós de partidas nos materiais moídos.

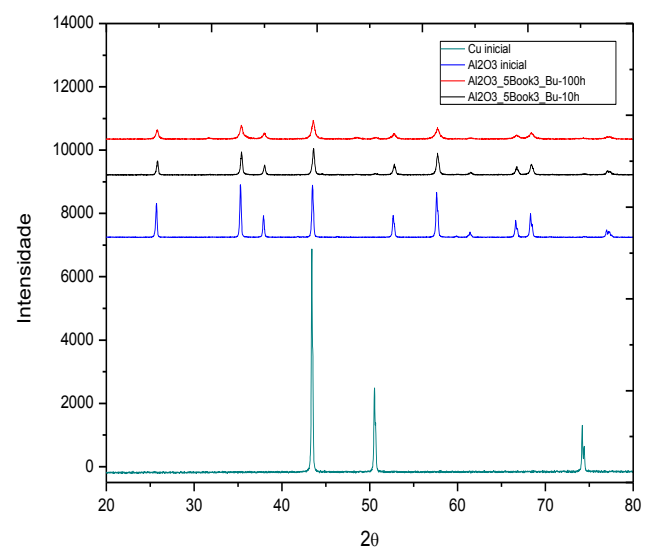

Figura 2. $\mathrm{DRX}$ dos pós iniciais de $\mathrm{Al}_{2} \mathrm{O}_{3}$ e $\mathrm{Cu}$ e $\mathrm{Al}_{2} \mathrm{O}_{3}-5 \% \mathrm{Cu} 10$ em 100 horas de moagem.

Nas Figuras 3 e 4 houve uma diferença de picos, visto na diminuição da cristalinidade do material à medida que se aumentou o tempo do processo de

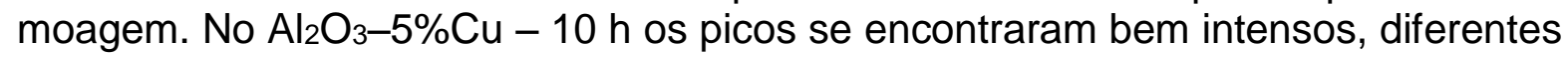
dos picos advindos do $\mathrm{Al}_{2} \mathrm{O}_{3}-5 \% \mathrm{Cu}-100 \mathrm{~h}$ que se mostraram menos intensos, com a base mais alargada, com uma leve indicação de amortização do material. Para Ying e Zhang [12], em seu trabalho sobre este compósito Cu-20 vol\% $\mathrm{Al}_{2} \mathrm{O}_{3}$, os picos da $\mathrm{Al}_{2} \mathrm{O}_{3}$ foram claramente detectados, além dos picos de Cobre.

\footnotetext{
* Contribuição técnica ao 69 Congresso Anual da ABM - Internacional e ao 14ํNNET - Encontro Nacional de Estudantes de Engenharia Metalúrgica, de Materiais e de Minas, 21 a 25 de julho de 2014, São Paulo, SP, Brasil.
} 


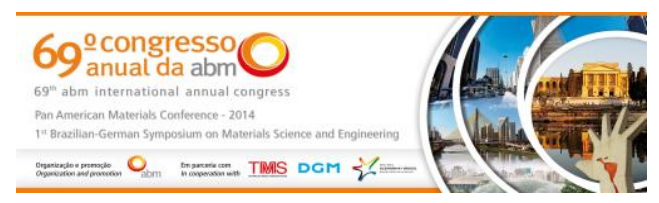

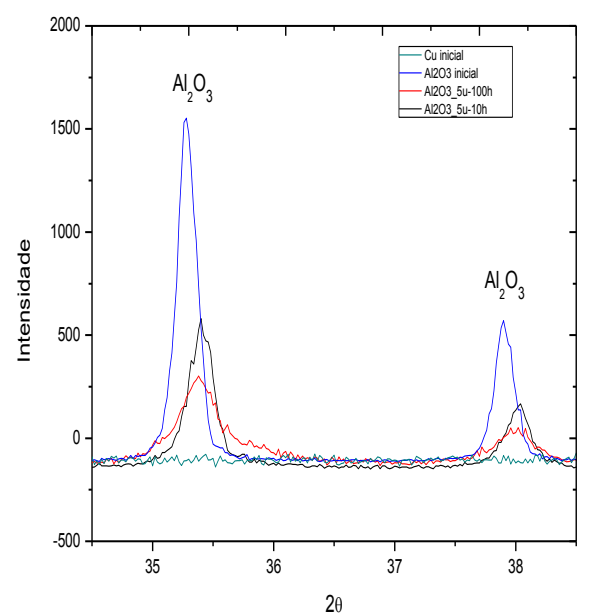

Figura 3. $\mathrm{DRX}$ do $\mathrm{Al}_{2} \mathrm{O}_{3}-5 \% \mathrm{Cu}, 10$ e $100 \mathrm{~h}$, em comparativo com a Alumina.
${ }^{\circ} \mathrm{Cu}$

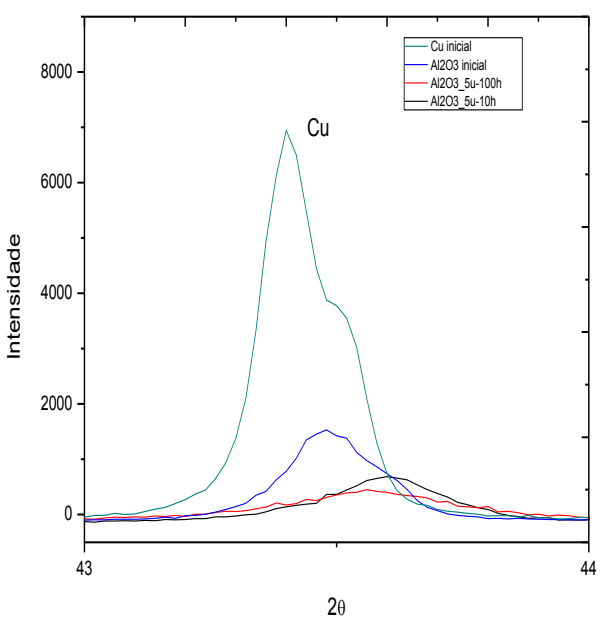

Figura 4. $\mathrm{DRX}$ do $\mathrm{Al}_{2} \mathrm{O}_{3}-5 \% \mathrm{Cu} 10 \mathrm{e}$ $100 \mathrm{~h}$, em comparativo com o Cobre.

De acordo com os dados granulométricos vistos na Figura 5, pode-se observar que o diâmetro médio do pó moído por 10 horas foi de 2,38 um, valor este que foi maior que o pó moído a 100 horas como sendo de 1,61um. Segundo Carvalho [13] confirma o referido resultado, quando remete que a medida que o tempo de moagem aumenta, as partículas tornam-se menores.

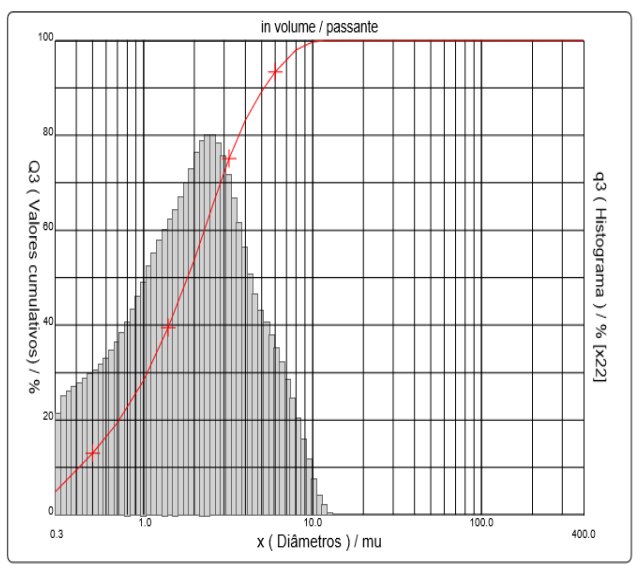

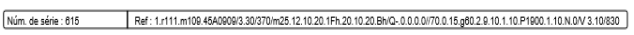

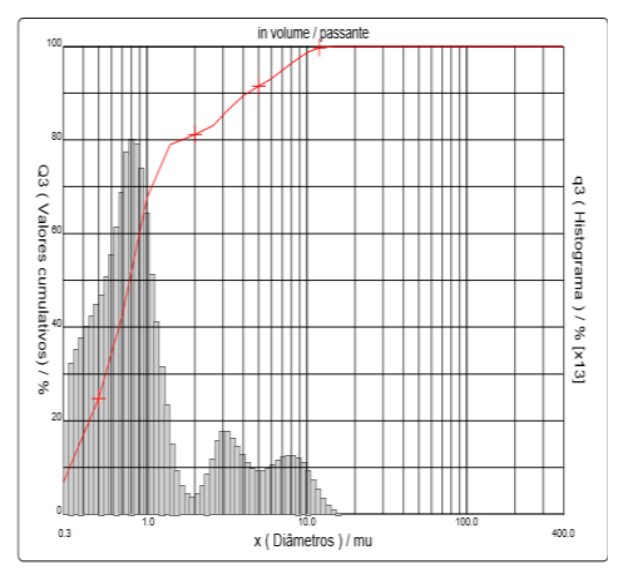

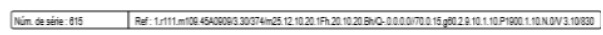

Figura 5. Distribuição do tamanho de partícula do pó moído do compósito $\mathrm{Al}_{2} \mathrm{O}_{3}-5 \% \mathrm{Cu}$, há 10 horas (esquerda) e 100 horas (Direita).

Quanto a Microscopia eletrônica de varredura, inicialmente pode-se realizar o mapeamento das amostras referentes aos pós de partidas $\left(\mathrm{Al}_{2} \mathrm{O}_{3}\right.$ e $\mathrm{Cu}$ ), como visualizado nas Figuras 6 e 7. Nos pós iniciais, foi detectado sua distribuição, a as formas dos grãos. Na alumina tem-se grãos pequenos (Figura 6), mais finos e no Cobre (Figura 7) pode-se visualizar grãos maiores.

* Contribuição técnica ao 69ํ Congresso Anual da ABM - Internacional e ao 14ํㅡㄹ ENEMET - Encontro Nacional de Estudantes de Engenharia Metalúrgica, de Materiais e de Minas, 21 a 25 de julho de 2014, São Paulo, SP, Brasil. 


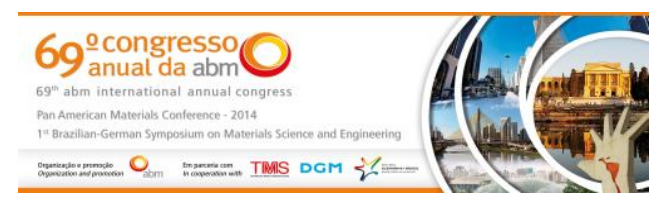

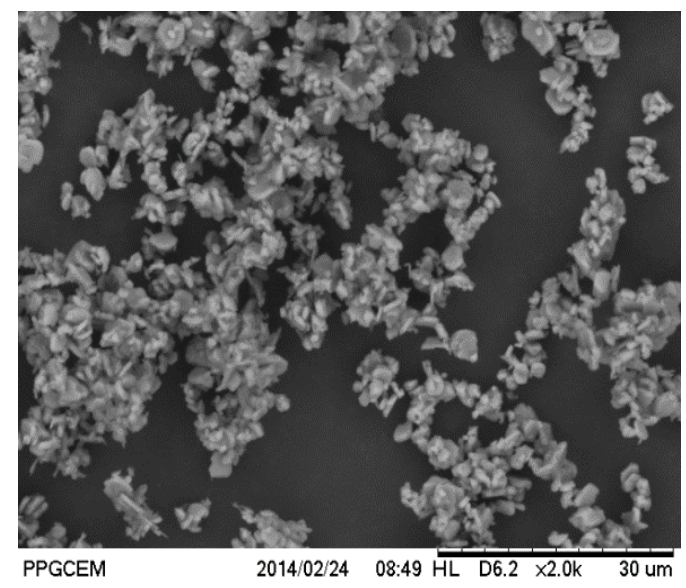

Figura 6. $\mathrm{MEV}$ do pó puro inicial de $\mathrm{Al}_{2} \mathrm{O}_{3}$ (Alumina).

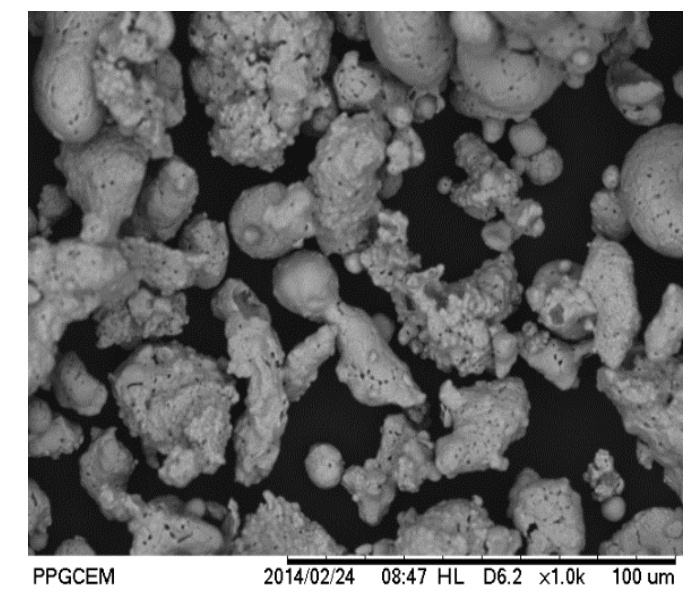

Figura 7. MEV do pó puro inicial do $\mathrm{Cu}$ (Cobre).

Nós pós submetidos ao processo de moagem de alta energia, pode-se ver a distribuição das partículas de $\mathrm{Al}_{2} \mathrm{O}_{3}$ na matriz metálica das amostras do pó compósito $\mathrm{Al}_{2} \mathrm{O}_{3}-5 \% \mathrm{Cu}$. Nas figuras 8 e 9 pode-se visualizar a distribuição das fases matriz-cerâmica, a cor escura é da cerâmica $\mathrm{Al}_{2} \mathrm{O}_{3}$, e a cor cinza clara é referente a matriz de $\mathrm{Cu}$. Estudos realizados por In-Young Ko, 2010, em seu trabalho sobre propriedades e consolidação do compósito $\mathrm{Al}_{2} \mathrm{O}_{3}-\mathrm{Cu}$, justifica a presença das fases com percepção e identificação da matriz metálica e do reforço cerâmico.

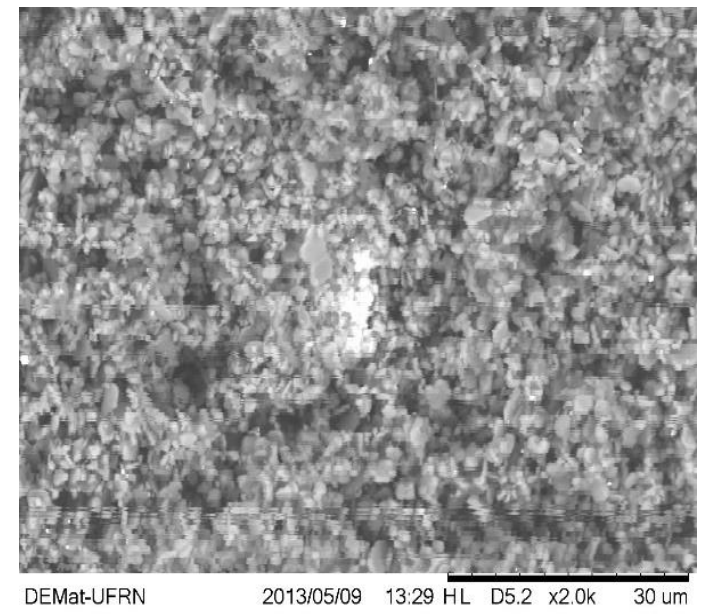

Figura 8. $\mathrm{MEV}$ do $\mathrm{Al}_{2} \mathrm{O}_{3}-5 \%$ por 10 horas de moagem 2000X.

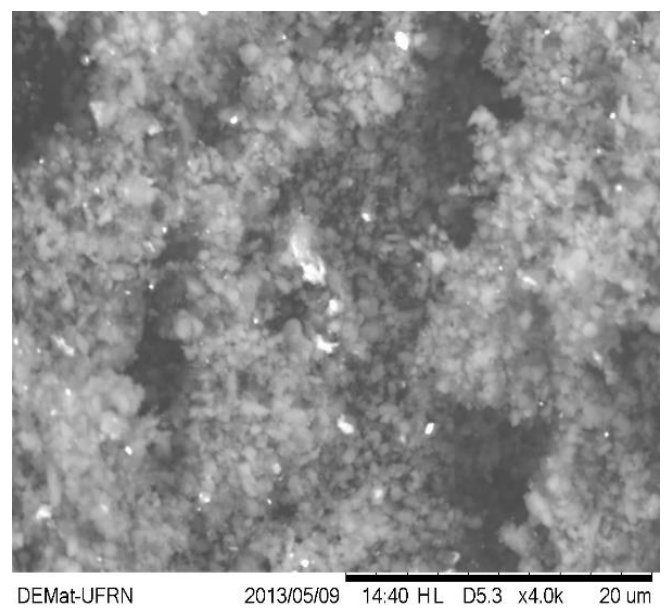

Figura 9. $\mathrm{MEV}$ do $\mathrm{Al}_{2} \mathrm{O}_{3}-5 \%$ por 100 horas de moagem $4000 \mathrm{X}$.

Nas Figuras 10 e 11, pode-se perceber a dispersão das partículas cerâmicas em meio a matriz de Cobre das amostras sinterizadas, similarmente observado por Ying, et al Zhang, 2000, em que afirma que as regiões escuras são a forma das partículas compostas pelo oxido cerâmico. As zonas claras são a fase de cobre puro. É provável que a fase de cobre puro nos espaços entre as regiões dos compósitos foi causada pelo fluxo preferencial do cobre durante a sinterização.

* Contribuição técnica ao 69 Congresso Anual da ABM - Internacional e ao 14ํㅡㄹ ENET - Encontro Nacional de Estudantes de Engenharia Metalúrgica, de Materiais e de Minas, 21 a 25 de julho de 2014, São Paulo, SP, Brasil. 

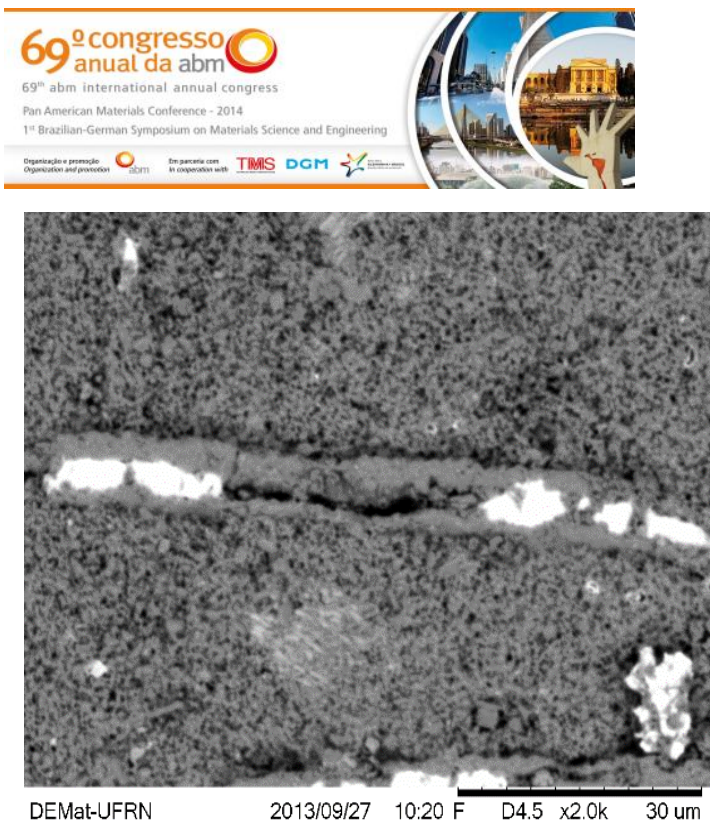

Figura 10. $\mathrm{MEV}$ do $\mathrm{Al}_{2} \mathrm{O}_{3}-5 \% \mathrm{Cu}$ sinterizado (400x).

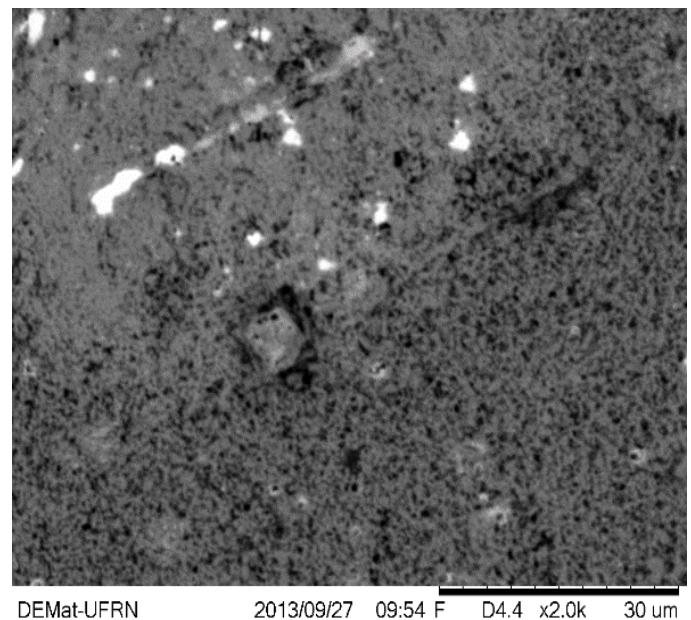

Figura 11. $\mathrm{MEV}$ do $\mathrm{Al}_{2} \mathrm{O}_{3}-5 \% \mathrm{Cu}$ sinterizado (2000x).

As amostras foram sinterizadas a $1300^{\circ} \mathrm{C}$, contudo sabe-se que o ponto de fusão da alumina é $2050^{\circ} \mathrm{C}$ e o Cobre exibe como temperatura de fusão $1084,87^{\circ} \mathrm{C}$, logo ocorrerá o surgimento de uma fase liquida em meio a sinterização, já que o PF do Cobre está abaixo da temperatura de sinterização. Desta forma, como a quantidade de cobre foi pequena em relação as partículas da cerâmica, houve o espalhamento do líquido recém formado ao redor das partículas sólidas que conduzem ao rearranjo dessas partículas, a densificação da estrutura, e a consequente permanência da forma do compactado ao final da sinterização. Neste contexto, PRICE 1938, descreve que a densificação via sinterização com fase liquida geralmente requer transporte de quantidade substancial de sólido dissolvido através do liquido e a quantidade de fase liquida deve ser pequena o suficiente para que o compacto mantenha sua forma durante a sinterização. Quanto a análise da densidade, como foram obtidos duas amostras $\mathrm{Al}_{2} \mathrm{O}_{3}-5 \% \mathrm{Cu}$, calculou-se a média das densidades reais, tanto das amostras verdes (compactadas) como das amostras sinterizadas, como pode ser visto na Tabela 1.

Tabela 1. Tabela das densidades relativas das amostras compactadas e sinterizadas

\begin{tabular}{lc}
\hline Amostra & Densidade Relativa (\%) \\
\hline Compactado & 0,26 \\
\hline Sinterizado & 0,31 \\
\hline Fonte: $L M C M E-$ UFRN &
\end{tabular}

A densidade relativa do compactado foi menor que a da amostra sintetizada, mostrando que houve densificação do material. Como a sinterização ocorreu em fase liquida, a tendência do liquido é cobrir as partículas sólidas eliminando a interface sólido-vapor, com mais rapidez que no estado sólido, promovendo um aumento do percentual de densidade do material [17]. 1985 confirma que a presença do líquido é responsável pela rapidez e percentual de densificação da estrutura.

* Contribuição técnica ao 69 Congresso Anual da ABM - Internacional e ao 14ํㅡㄹ ENET - Encontro Nacional de Estudantes de Engenharia Metalúrgica, de Materiais e de Minas, 21 a 25 de julho de 2014, São Paulo, SP, Brasil. 


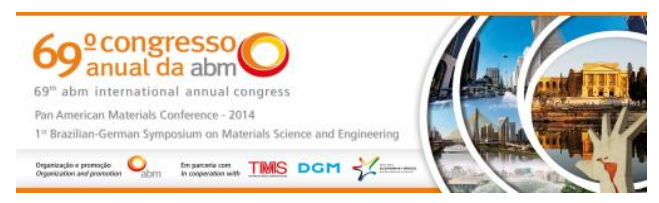

\section{CONCLUSÃo}

A moagem de alta energia em conjunto com o processo de metalurgia do pó tradicional é uma técnica eficaz para a produção do compósito $\mathrm{Al}_{2} \mathrm{O}_{3}-5 \% \mathrm{Cu}$

Cerâmico matriz metálica. O tempo de moagem interfere na cristalinidade do material, uma vez que quanto maior o tempo de moagem, maior será o impacto das bolas com o material promovendo assim defeitos na estrutura do compósito. Além disso, as partículas que obtiveram o menor diâmetro médio foi as partículas moídas a 100 horas. Houve uma maior dispersão das partículas na matriz. A densificação do material final se mostrou eficaz com um percentual de 0,31 de densificação do material sinterizado, comprovando assim a eficiência de síntese do processo de moagem e sinterização na consolidação do compósito $\mathrm{Al}_{2} \mathrm{O}_{3}-5 \% \mathrm{Cu}$.

\section{Agradecimentos}

Agradecemos ao DEMat-UFRN pelo apoio concedido ás analises realizadas, ao LMCME (Laboratório de Materiais cerâmicos e Metais especiais) pelo espaço outorgado para a realização dos experimentos.

\section{REFERÊNCIAS}

1 Callister WD, Ciência e Engenharia de Materiais: Uma Introdução. 5ª Edição, Editora LTC, 2002.

2 Tjong SC, Ma ZY, Mater. Sci. Eng, 2000; 29: 49-113.

3 Kruis FE, Fissan, Kona H. 1999;17:130-9.

4 Niihara K, Nakahira A. Particulatestrengthened ceramics nanocomposites. In: Vincenzini, P. (Ed.). Advanced Structural Inorganic Composites.Elsevier, p. 637, 1991.

5 Celebi EG, Yener T, Altinsoy I, Ipek M, Zyetin S, Bindal C.,J. Alloys Compd, 2011; 509: 6036-6042.

6 Motta MS, Jena PK, Brocchi EA, Solorzano IG, Mater. Sci. Eng. C, 2001; 15: 175-177.

7 Friedrich K, Engineering Materials Handbook, vol. 4-Ceramics and Glasses (ASM, Materials Park, 1991).

8 Ashby MF, Jones DRH, Engineering Materials 1 (International Series on Materials Science and Technology), vol. 34 (Pergamon, Oxford, 1986).

9 Cullity BD. Elements of x-ray Difraction, 2 edição, Addison-Wesley publishing Company, Canada, 1978: 284-285.

10 Chinelatto ASA. Evolução Microestrutural Durante a Sinterização de Pós Finos e de Alta Pureza de Alumina [Tese Doutorado em Ciência e Engenharia de Materiais]. São Carlos: Universidade Federal de São Carlos, 2002.

11 Motta MS, Brocchi EA, Jena PK. Síntese Por Redução Preferencial E Caracterização Microestrutural Do Nano-Compósito $\mathrm{Cu}-\mathrm{Al}_{2} \mathrm{O}_{3 . .}$ Rio de Janeiro, (2003).

12 Ying DY, Zhang DL. Processing of $\mathrm{Cu} \mathrm{Al} 2 \mathrm{O} 3$ metal matrix nanocomposite materials by using high energy ball milling. Materials Science and Engineering, 2000: 152-156, New Zealand.

13 Carvalho WM. Estudo da moagem de alta energia e sinterização de pós compósitos WCu [Dissertação de Mestrado]. PPGCEM/UFRN; 2008.

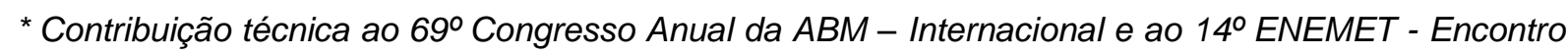
Nacional de Estudantes de Engenharia Metalúrgica, de Materiais e de Minas, 21 a 25 de julho de 2014, São Paulo, SP, Brasil. 
14 Yong K I, Kim NR, Lee JY, Park NR, Doh JN, Shon IJ. Properties and consolidation of nanocrystalline 3Cu-Al2O3 composite by rapid sintering. Springer Science+Business Media B.V., 2010.

15 Suryanarayana, C. Mechanical alloying and milling. Progress in Materials Science. 2001;46:1-184.

16 Price GHS, Smithells CJ, Williams SV. Smtered alloys part I: copper- nickel-tungsten a Boys sintered which a liquid phase present. J. of the Inst, of Metals, 1938; 62: 239-64.

17 German RM. Liquid Phase Sintering. In: German RM. New York, NY: 1985, cap.7, p.160-163.

* Contribuição técnica ao 69 Congresso Anual da ABM - Internacional e ao 14ํNEMET - Encontro Nacional de Estudantes de Engenharia Metalúrgica, de Materiais e de Minas, 21 a 25 de julho de 2014, São Paulo, SP, Brasil. 